\title{
Heat transfer of droplets impinging onto a wall above the Leidenfrost temperature
}

\section{Transferts thermiques lors de l'impact de goutte sur une paroi en régime de Leidenfrost}

\author{
Pierre Dunand $^{\mathrm{a}, \mathrm{b}}$, Guillaume Castanet ${ }^{\mathrm{a}, \mathrm{b}, *}$, Michel Gradeck ${ }^{\mathrm{a}, \mathrm{b}}$, Fabrice Lemoine ${ }^{\mathrm{a}, \mathrm{b}}$, \\ Denis Maillet ${ }^{\mathrm{a}, \mathrm{b}}$ \\ a Université de Lorraine, LEMTA, UMR 7563, 54500 Vandoeuvre-lès-Nancy, France \\ b CNRS, LEMTA, UMR 7563, 54500 Vandoeuvre-lès-Nancy, France
}

\section{A R T I C L E I N F O}

Keywords:

Droplet impact

Hot wall

Leidenfrost

Laser-induced fluorescence

Heat transfer

\section{Mots-clés:}

Impact de gouttes

Paroi chaude

Leidenfrost

Fluorescence induite par Laser

Transferts thermiques

\begin{abstract}
A B S T R A C T
In aero-engines, droplet/wall interaction phenomena have a considerable influence on the mixture formation process and on wall heat fluxes. Impinging droplets may rebound, splash into secondary droplets or form a liquid film onto the solid surface. Droplet rebound and splashing is also a mechanism for the back penetration of the fuel vapor in the central region of the combustion chamber where the gas temperature is high enough for ignition. This work is an experimental study aiming at characterizing the heat transfers induced by the impingement of water droplets (diameter $80-180 \mu \mathrm{m}$ ) on a thin nickel plate heated by electromagnetic induction. The temperature of the rear face of the nickel sample is measured by means of an infrared camera and the heat removed from the wall due to the presence of the droplets is estimated using a semi-analytical inverse heat conduction model. In parallel, the temperature of the droplets is measured using the two-color LaserInduced Fluorescence Thermometry which has been extended to imagery for the purpose of these experiments. The measurements of the variation in the droplet temperature occurring during an impact allow determining the liquid sensible heat. Measurements are performed at surface conditions well above the Leidenfrost temperature. A wide range of Weber numbers corresponding to the bouncing and splashing regimes are tested. Comparison between the heat flux removed from the wall and the sensible heat gained by the liquid allows estimating the heat flux related to liquid evaporation. Results reveal that the respective level of the droplet sensible heat and the heat lost due to liquid vaporization can vary significantly with the droplet sizes and the Weber number.
\end{abstract}

\section{R É S U M É}

Les phénomènes d'interactions gouttes/paroi jouent un rôle important dans les chambres de combustion des turboréacteurs. Ils participent notamment à la production et au mélange de la vapeur de combustible ainsi qu'aux transferts de chaleur en paroi. Différents régimes d'impact peuvent être observés selon les conditions: le rebond, l'éclatement avec la formation de gouttes secondaires ainsi que le dépôt d'un film liquide. La vapeur générée lors de ces interactions migre ensuite vers l'intérieur de la chambre de combustion, où la température des gaz est suffisamment élevée pour permettre l'inflammation. Cette étude expérimentale a pour objectif de caractériser les transferts thermiques lors de l'impact de

\footnotetext{
* Corresponding author at: Université de Lorraine, LEMTA, UMR 7563, 54500 Vandoeuvre-lès-Nancy, France.

E-mail address: guillaume.castanet@univ-lorraine.fr (G. Castanet).
} 
gouttelettes d'eau sur une pastille de Nickel chauffée par induction électromagnétique. La température face-arrière de l'échantillon de nickel est mesurée à l'aide d'une caméra infrarouge, et le flux extrait à la paroi est estimé par un modèle d'inversion semi-analytique tenant compte de la conduction dans la pastille ainsi que des pertes convective et radiative. En parallèle, l'échauffement des gouttes est caractérisé à l'aide de la fluorescence induite par laser à deux couleurs. Cette technique a été récemment étendue à de l'imagerie dans le cadre de cette étude. Elle permet d'estimer la chaleur sensible gagnée par les gouttes lors de l'impact. La paroi est maintenue à une température supérieure à la température de Leidenfrost, tandis que le nombre de Weber des gouttes incidentes est modifié de manière à obtenir des rebonds et des éclatements. La comparaison entre la chaleur sensible gagnée par les gouttes et le flux extrait à la paroi permet finalement d'évaluer le flux de chaleur prélevée par évaporation. Les contributions au refroidissement de ces flux varient de manière significative en fonction du diamètre des gouttes, mais aussi du nombre de Weber des gouttes incidentes.

\section{Nomenclature}

$a$

Cp heat capacity $\left[\mathrm{kJ} \mathrm{kg}^{-1} \mathrm{~K}^{-1}\right]$

$D \quad$ droplet diameter [m]

$f \quad$ injection frequency [hz]

$I_{0} \quad$ laser intensity [W]

$K \quad$ Mundo number

$L_{v} \quad$ latent heat $[\mathrm{kJ} / \mathrm{kg}]$

$m \quad$ droplet mass [kg]

Oh Ohnesorge number

$p \quad$ Laplace parameter

$Q_{l} \quad$ sensible heat gained by the liquid [J]

$R_{0} \quad$ reference ratio

$R_{f} \quad$ fluorescence ratio

$T$ temperature [K]

$V \quad$ measurement volume

$V_{d} \quad$ droplet velocity $[\mathrm{m} / \mathrm{s}]$

$V_{n} \quad$ normal droplet velocity [m/s]

$W \quad$ transmittance

We Weber number

$Z \quad$ impedance $\left[\mathrm{Km} \mathrm{W}^{-1}\right]$

Symbols

$\alpha \quad$ droplet incidence angle $\left[{ }^{\circ}\right]$ or eigenvalues

$\gamma \quad$ surface tension [N m] $\delta \quad$ Dirac distribution or Kronecker symbol

$\varepsilon \quad$ cooling efficiency

$\lambda$ wavelength [nm] or thermal conductivity [W m ${ }^{-1} \mathrm{~K}^{-1}$ ]

$\mu \quad$ dynamic viscosity [Pas]

$\rho \quad$ density $\left[\mathrm{kg} / \mathrm{m}^{3}\right]$

$\Phi_{w} \quad$ heat removed from the wall by a single droplet [J]

$\Phi_{\text {vap }} \quad$ heat taken by the evaporation [J]

\section{Introduction}

In aero-engines, droplet/wall interaction phenomena have a considerable influence on the mixture formation process and on wall heat fluxes. Impinging droplets may rebound, splash into secondary droplets or form a liquid film onto the solid surface [1]. Droplet rebound and splashing is also a mechanism for the back penetration of the fuel vapor in the central region of the combustion chamber where the gas temperature is high enough for ignition. Droplet/wall interactions can also be found in cooling applications, which require a high heat dissipation rate. Spray quenching is very efficient compared to other cooling techniques. The main reason is that vapor can easily escape even if the temperature of the wall is well above the Leidenfrost temperature. However an optimization of these applications needs a complete understanding of the complex fluid flow and heat transfer characteristics when sprays interact with hot surfaces. The modeling of each physical phenomenon occurring in the combustion chamber is required to improve the accuracy of existing numerical codes. Due to the complexity of a dense spray, elementary phenomena are studied as basic experiments using monosized jets (droplets having all the same size and velocity and being regularly spaced).

The impingement of droplets on solid surfaces has received a considerable attention throughout the decades. Many studies have been focused on the impact conditions that can occur at high or low droplet velocity, with deep or shallow 
liquid film, on hot or cold solid surfaces. Depending on these conditions, different behaviors can occur: the drops can spread over the solid surface, they can splash by creating a crown and droplet rebounds are also possible particularly on hot surfaces above the Leidenfrost temperature. Extensive experimental investigations were carried out to determine the parameters influencing the behavior of a single drop impact in order to characterize their respective influence. Some of these parameters describe the geometry and the dynamic of the drops [2,3], some refer to the physical properties of the liquid [2,4] or the solid surface [5]. Correlations based on dimensionless numbers characterizing the relative magnitude of the forces acting on the impinging droplet and estimated with the physical properties of the liquid before impact, i.e. Reynolds, Weber and Ohnesorge numbers [6] have been found.

This study concerns the non wetting conditions observed when the wall temperature is above the Leidenfrost temperature, which corresponds to the film boiling regime. A thin vapor layer forms quasi-instantaneously between the droplet and the wall and prevents the droplet to wet the wall. The splashing and the rebound of the droplet are the only behavior that can occur, depending on the size of the droplet and its normal component of velocity. An increase of the Weber number promotes the splashing [1]. We have firstly focused on the film boiling regime where the vapor cushion formed between the droplet and the solid insulates the droplet from the hot sample and thus limits drastically the heat transfer [7].

Heat transfers were generally characterized using thermocouples embedded in the sample thickness. It has been possible to monitor the history of the surface temperature at the location of the droplet impingement [8]. The heat flux to the wall was also estimated when the experimental set-up was designed to ensure a one-dimensional heat flux along the instrumented section containing a set of thermocouples. Advanced methods are even less intrusive and involve infrared thermography. Nevertheless, if measurements related to the wall provide valuable data to quantify the efficiency of the cooling, they have only a limited interest when focusing on the heat transfer occurring within the liquid phase. In particular, questions remain concerning the respective level of the droplet sensible heat variation and the heat lost due to liquid vaporization. To address this issue, it is necessary to use different measurement techniques. An inherent difficulty is the estimation of the mass of liquid evaporated during the droplet/wall interaction. To the best of our knowledge, few experimental data can be found in the literature for the mass loss during the impact in the splashing regime. In the bouncing regime, the ratio between outgoing and impinging liquid mass was evaluated by Le Clercq [9], using phase doppler measurements (PDA) and digital image processing. Although a correlation is provided by Le Clercq [9] and Rosa et al. [10] based on these measurement data, the accuracy of these direct measurements is questionable. The outcoming droplets are strongly deformed after their impingement and an accurate measurement of their volume or their mass is a real challenge with any optical method. According to [9] and [10], the variation in mass due to evaporation is rather important, about $25 \%$ for most of the rebound. This largely overestimates the experimental values by Watchers and Westerling [11] that were obtained using a completely different approach. Watchers and Westerling [11] collected the vapor expelled by the droplets and measured the mass of this vapor after condensation. The vapor was found to represent generally between 2 and $5 \%$ percent of the initial liquid mass.

Presently, a quite different strategy has been undertaken. The evaporation (i.e. the change in droplet size) is not directly measured but it is estimated indirectly from the application of energy conservation. All the heat fluxes involved in the cooling (namely the sensible heating of the liquid and the heat extracted from the wall) are measured except the heat flux associated with evaporation.

This article is an experimental study involving several measurement techniques for characterizing the heat transfer within the liquid phase and at the wall to be cooled. The temperature variation of the droplet is measured using a new optical technique: the two-color laser induced fluorescence (2CLIF) thermometry. In addition, an infrared camera measures the temperature field in the rear face of a thin nickel plate. A semi-analytical inverse heat conduction model allows estimating the heat flux on the front face of the plate where the droplets impinge. Finally, the heat flux lost by the wall and due to droplet/wall interaction is compared to the sensible heat stored in the outgoing droplets. This allows estimating the heat flux associated with evaporation of the drops.

\section{Droplet generation and experimental set-up}

In order to study droplet/wall interactions, an experimental set-up was specifically designed. A sketch of the experimental set-up is shown in Fig. 1. A linear monodisperse droplet stream is generated by the disintegration of a cylindrical liquid jet. The breakup is driven by a Rayleigh-type instability that can be triggered by mechanical vibrations using a piezoceramic. For some specific frequencies of the vibrations, the liquid jet split into equally spaced and monosized droplets. The size of the injector orifice and the inlet pressure can be changed from an experiment to another, which allows to some extents adjusting separately the diameter $D_{d}$, the frequency $f$ and the velocity $V$ of the droplets. In this study, the droplets range from $80 \mu \mathrm{m}$ to $250 \mu \mathrm{m}$ while their velocity is of the order of a few $\mathrm{m} / \mathrm{s}$. The droplet generator can be rotated precisely to any prescribed angle $\alpha$ of incidence. The temperature of the injector body is regulated while the liquid temperature is measured by a thermocouple placed just before the outlet of the injector. Water droplets impact periodically a thin disk of nickel (thickness is $500 \mu \mathrm{m}$ and radius $R=12.5 \mathrm{~mm}$ ) which is heated using an electromagnetic inductor device. In this contactless heating technique, the distribution of the heat sources in the skin depth of the metallic sample is perfectly controlled. The low thickness of the nickel disc allows limiting the damping of the thermal response at the rear face (side of the sample opposite to the droplet impact). The upper surface of the nickel on which the droplets are impacting, is polished so as to obtain a very smooth surface. The oxidation of nickel occurs easily when the wall temperature exceeds $500^{\circ} \mathrm{C}$. 


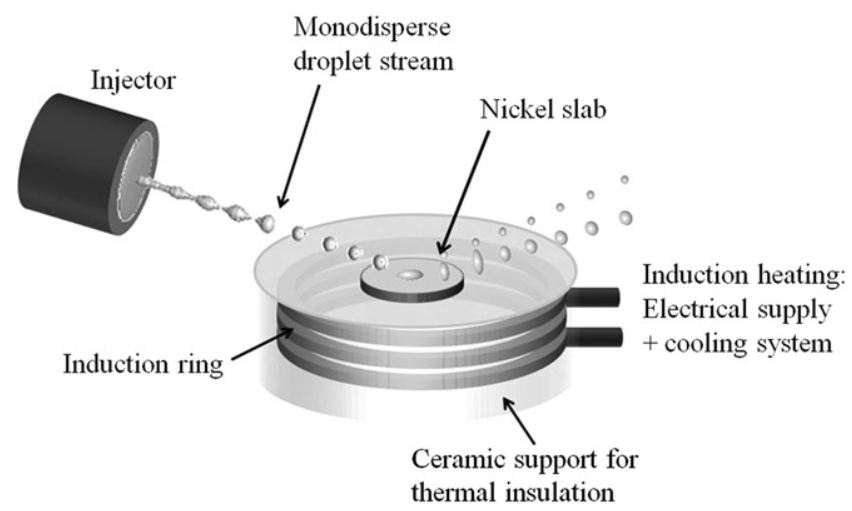

Fig. 1. Experimental set-up.

An oxide layer covers the surface of the nickel disk. This green and gray layer of nickel oxide that covers the disc is very stable, and did not provide a significant change of the overall roughness. In addition, the oxidation increases the radiative emissivity of the wall.

\section{Shadowgraphy for measuring droplets size and velocity}

A high-speed (HS) camera is used to visualize the shape of the droplet stream impinging onto the heated wall. The HS camera is a Phantom v710 equipped with a 12-bits CMOS sensor that can provide up to 7500 fps at full resolution $(1280 \times 800$ pixels). It is used with a reduced resolution to perform the image acquisition at a much higher frame rate, typically in the order of $100,000 \mathrm{fps}$. This is sufficient to resolve in time the droplet/wall interactions in the experimental conditions encountered in this study. The droplets are illuminated from behind using a very bright light source (a $400 \mathrm{~W}$ HMI lamp with a parabolic reflector). A zoom lens allows observing in detail the droplet deformation and possible splashing, with a field of view ranging from $400 \mu \mathrm{m}$ to $3 \mathrm{~mm}$. The images are then processed with a homemade detection and tracking program in order to determine the main features of the impact. A genetic algorithm is used for the tracking of the particle trajectories. It is based on the multi-hypothesis tracking method proposed by Reid [12]. Boulesteix [13] describes an adaption of this method for the tracking of droplets. Joint distributions of the droplets size and velocity can be derived from the processing of the images. Other important parameters such as the incident angle, the normal and tangential velocities, the residence time, or the spreading diameter of the droplets can be also determined.

\section{Two-color laser induced fluorescence for measuring the droplet temperature}

Different optical techniques have been developed in the past decades to characterize the droplet temperature. However, for some methods like the rainbow refractometry, a perfect spherical shape of the droplet is required to obtain a correct temperature after inversion. The two-color laser induced fluorescence (2cLIF) has already demonstrated its ability to characterize the temperature of droplets in various situations including droplet evaporation in either inert or reactive flows [14, 15]. The technique was also used to determine the droplet change in temperature during their impingement onto a heated solid surface [16]. In this study, the 2cLIF thermometry was restricted to point-wise measurements, which imply a cumbersome point-by-point scanning to reconstruct the temperature distribution in the liquid phase of the flow. More recently, the technique was extended to planar laser induced fluorescence (PLIF) in order to obtain the temperature field [17].

The 2cLIF thermometry is based on the measurement of the fluorescence intensity of a single dye tracer. In liquids, the fluorescence quantum yield is strongly influenced by the quenching, which depends on the temperature. When a laser beam induced the fluorescence of a dye dissolved into a liquid medium, the fluorescence signal I emerging from the medium toward the detector can be expressed as:

$$
I_{f}(\lambda)=K_{\text {opt }}(\lambda) K_{\text {spec }}(\lambda) I_{0} c V \exp \left(\frac{\beta(\lambda)}{T}\right)
$$

where $K_{\text {opt }}$ is a parameter depending on the optical properties of the detection system (e.g. the solid angle of the detection, the spectral sensitivity of the detectors), $K_{\text {spec }}$ is a parameter depending on the spectroscopic properties of the tracer in its solvent. $c$ is the concentration in dye molecules and the product $c . V$ corresponds to the number of molecules that are illuminated by the laser beam while in the field of view of the detector. $I_{0}$ is the intensity of the laser beam before crossing the absorbing medium. In Eq. (1), it is implicitly assumed that the absorption of the laser beam and the fluorescence can be neglected along the ray path in the liquid medium. Fig. 2 shows the evolution of the fluorescence emission spectrum of Rhodamine 640 ( $\mathrm{Rh} 640, \mathrm{C}_{32} \mathrm{H}_{31} \mathrm{~N}_{2} \mathrm{O}_{3} \cdot \mathrm{ClO}_{4}$, also called rhodamine 101) that has been selected for this study. A decrease in the fluorescence intensity is clearly observed when increasing the temperature. The evolution of $\beta(\lambda)$ is also superimposed 


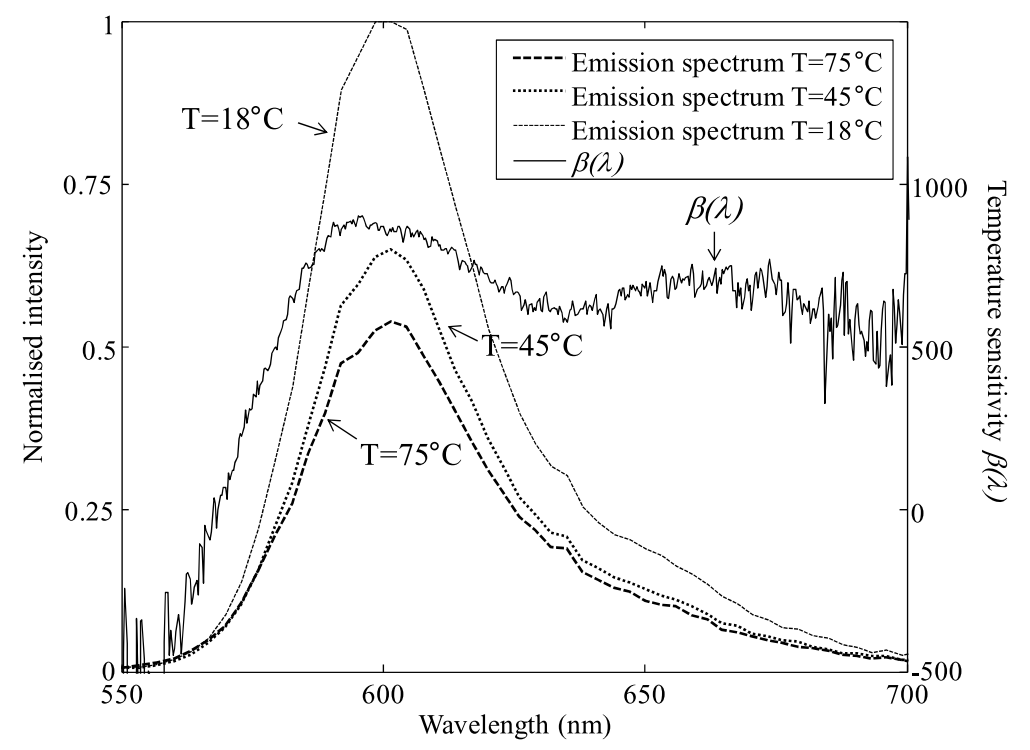

Fig. 2. Fluorescence spectrum of Rh640 dissolved in water and the wavelength dependence of $\beta(\lambda)$.

in the figure. The value $\beta(\lambda)$ varies significantly between the right and the left sides of the spectrum, meaning that the effect of temperature is not the same for all the wavelengths in the fluorescence spectrum. Optical filters are used in the measurement to select specific spectral bands of the fluorescence emission. For a given spectral band $i$, the fluorescence intensity $I_{f}(\lambda)$ is integrated over the wavelength range $\left[\lambda_{i, 1}, \lambda_{i, 2}\right]$. Based on experimental measurements, Lavieille et al. [18] suggest approximating this expression as:

$$
I_{f, i}=\int_{\lambda_{i, 1}}^{\lambda_{i, 2}} I(\lambda) \mathrm{d} \lambda \approx K_{o p t, i} K_{s p e c, i} I_{l} c V \exp \left(\frac{A_{i}}{T^{2}}+\frac{B_{i}}{T}+C_{i}\right)
$$

In this expression, $A_{i}$ and $B_{i}$ are specific to a given combination of dye, solvent, excitation wavelength, and spectral band. In contrast, $C_{i}$ depends on the exact configuration of the experimental system and can change from one measurement configuration to another. The ratio $R_{f}$ of the intensities of two different spectral bands is given by:

$$
R_{f}=\frac{I_{f, 1}}{I_{f, 2}}=\frac{K_{o p t, 1} K_{\text {spec }, 1}}{K_{o p t, 2} K_{\text {spec }, 2}} \exp \left(\frac{A}{T^{2}}+\frac{B}{T}+C\right)
$$

where $X=X_{1}-X_{2}$ stands for $X=A, B$ or $C$. The ratio of the fluorescence intensity of two bands allows eliminating the effects of parameters that are unknown or difficult to control such as the variations in laser intensity, the tracer concentration or the measurement volume during the acquisitions. When the technique is applied in imagery, only $A$ and $B$ do not depend on the pixel position in the image. All other variables can change from one pixel to the other, especially the parameters $K_{o p t, i}, K_{\text {spec }, i}$ and $C$. Even under isothermal conditions, the fluorescence ratio is not necessarily uniform. To eliminate the influence of the detection system, a reference measurement at a known temperature $T_{0}$ (with the same optical configuration as for the measurement) is recorded. Denoting $R_{0}$ the fluorescence ratio obtained in the reference measurement, the temperature can be derived from:

$$
\ln \left(\frac{R_{f}}{R_{0}}\right)=A\left(\frac{1}{T^{2}}-\frac{1}{T_{0}^{2}}\right)+B\left(\frac{1}{T}-\frac{1}{T_{0}}\right)
$$

Once $A$ and $B$ are known, Eq. (4) can be used to determine the liquid temperature. These parameters are obtained by a calibration in a temperature-controlled cell using the full measurement system in particular for the selected spectral bands of detection. These bands correspond to the ranges [555-565 nm] and [635-685 nm] and are selected with regards to their intensity level as well as their sensitivity to the temperature (Fig. 2). The variation of the fluorescence of ratio is about $1.4 \% / \mathrm{K}$ which seems in practice enough to measure the droplet temperature with an accuracy of about $2{ }^{\circ} \mathrm{C}$.

The measurement system of the 2cPLIF technique is illustrated in Fig. 3. The excitation of Rh640 is achieved by means of a CW Nd:YAG laser (Laser Quantum Finesse, 6 W@532 nm). An arrangement of spherical and cylindrical lenses provides a laser sheet with a thickness of $220 \mu \mathrm{m}$ and a height of $16 \mathrm{~mm}$ in the measurement zone. This latter is observed by a Questar QM-1 long distance microscope, which is positioned at right angle at a working distance of about $84 \mathrm{~cm}$. The microscope field of view is then about $3.5 \times 3.5 \mathrm{~mm}^{2}$. A holographic filter (Notch Plus, Kayser Optical) is used to block the 


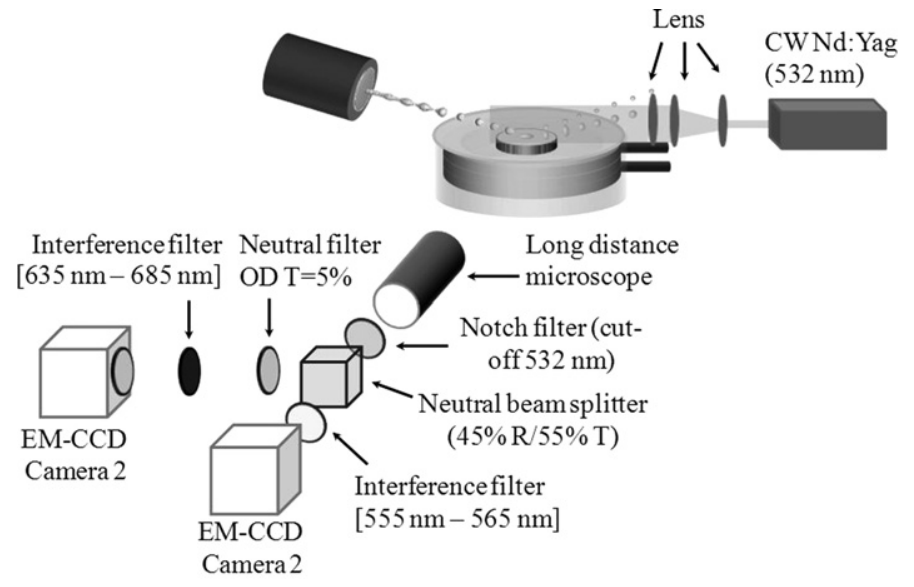

Fig. 3. Optical layout of the 2cPLIF technique.

a)

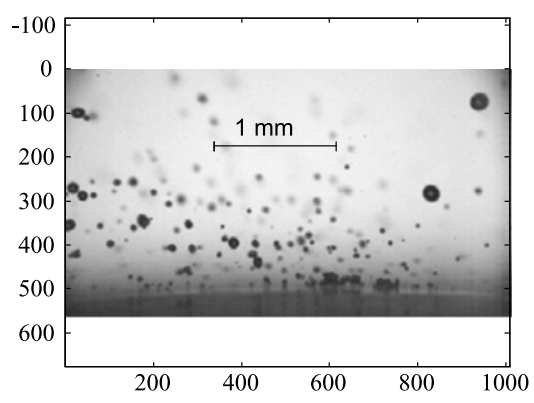

b)

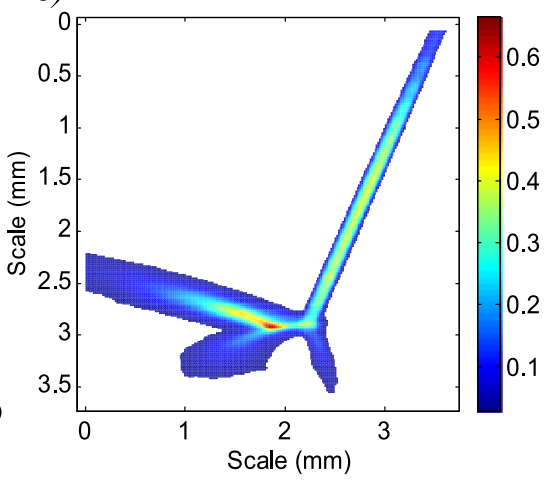

c)

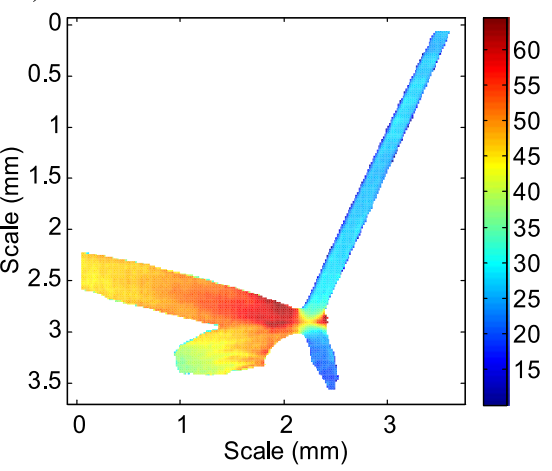

Fig. 4. An example of 2 CPLIF temperature measurement in the case of a splashing $\left(D=180 \mu \mathrm{m}, V_{d}=10 \mathrm{~m} / \mathrm{s}, \alpha=70.3^{\circ}, f=12 \mathrm{kHz}, T_{w}=540^{\circ} \mathrm{C}\right)(\mathrm{a}$ : image of the droplets by shadowgraphy without any correction of the distorsion, b: fluorescence intensity field, c: temperature distribution).

Mie scattering of the laser light at $532 \mathrm{~nm}$. A neutral beamsplitter ( $R / T$ 45/55\%) allows the separation of the fluorescence signal for its acquisitions by the cameras. Interference filters are mounted in front of the cameras and allows selecting the spectral bands mentioned before. Two electron-multiplying CCD cameras (Hamamatsu EM-CCD camera C9100-02) having 14 bits (16,384 levels) of intensity information with a spatial resolution of $1000 \times 1000$ pixels image the fluorescence field in the measurement zone. To improve the detection statistics, a $2 \times 2$ or $4 \times 4$ binning of the pixels is applied, even if the spatial resolution is reduced. Two images (one for each camera) are acquired simultaneously by using a common external trigger source. During the experiments, the cameras exposure time is set to a few tens of milliseconds to ensure a sufficient signal-to-noise ratio. Meanwhile, the droplets injected at about $10 \mathrm{kHz}$ cover several millimeters and their contribution to the fluorescence signal is cumulated. The images correspond finally to a time averaged fluorescence field (Fig. 4). The measurements were performed with moderate gains to limit the noise.

One of the main difficulties related to this experimental setup is the pixel-by-pixel correspondence of the camera images. The approach described in [17] is applied here. The least mean squares are used to find a combination of rotation and translation that minimizes the distance between the images of the fluorescence field taken by the two cameras. The solution obtained for one pair of images is generally optimal, i.e. it can be applied to another pair of images with a maximum error less than one pixel.

Finally, the measurements are performed with a concentration of Rh640 equal to $5 \times 10^{-5} \mathrm{~mol} / \mathrm{L}$. This concentration is relatively high and the reabsorption of the fluorescence within the droplet cannot be ignored. However, this high concentration is required to limit the effect of the droplet size and shape on the fluorescence spectrum which has been described by Labergue et al. [19]. In the same time, the application of the Beer law indicates that the fluorescence ratio $R_{f}$ is modified by $3 \%$ in the worth case (i.e. for an optical path length of $200 \mu \mathrm{m}$ ).

Fig. 4 shows an example of measurement in the case of a splashing. A significant heating of the droplet resulting from their impingement is observed. The fluorescence field is not uniform in an image; this is mainly related to the time averaged liquid concentration, which varies strongly in space. The liquid concentration is the more important near the impact region where the droplets are strongly squeezed before their break-up. From these images of the temperature field, the average temperature of primary and secondary droplets can be calculated. The average is weighted by the fluorescence 
intensity since this latter is roughly proportional to the liquid mass flow rate crossing the region of interest (ROI) during the integration time of the cameras.

$$
T_{m}=\iint_{R O I} T(x, y) I_{f}(x, y) \mathrm{d} x \mathrm{~d} y / \iint_{R O I} I_{f}(x, y) \mathrm{d} x \mathrm{~d} y
$$

\section{Infrared thermography and inverse conduction model for the wall heat flux estimation}

The temperature field of the Nickel slab was measured using a Cedip JADE III infrared camera. As the slab is impinged on one of its face by the droplet stream (it is noted "front face"), we chose to measure the temperature field on the other face (it is noted "rear face"). This camera is build around a focal plane array of photonic detectors working in the [3$5 \mu \mathrm{m}$ ] spectral interval. The IR camera is equipped with a narrow [3.97-4.01 $\mu \mathrm{m}$ ] filter. It allows the measurement of up $320 \times 240$ pixels/frame at sampling frequencies of the order of $60 \mathrm{~Hz}$. A specific inverse heat conduction model was thus developed to recover the unknown boundary on the front face namely the heat flux due to the impingement of the droplet stream (see the next section). This requires the analytical solution of the heat equation using integral transforms.

\subsection{Solution of the direct heat transfer problem}

One considers the internal transient conduction within a finite circular disk whose radius is $R=12.5 \mathrm{~mm}$ and thickness $e=500 \mu \mathrm{m}$. Assuming constant thermophysical properties, the basic set of equations are thus:

$$
\begin{aligned}
& \frac{\partial^{2} T}{\partial r^{2}}+\frac{1}{r} \frac{\partial T}{\partial r}+\frac{\partial^{2} T}{\partial z^{2}}=\frac{1}{a} \frac{\partial T}{\partial t} \\
& T(r, z, t=0)=T_{\text {init }}(r, z) \\
& -\left.\lambda \frac{\partial T}{\partial r}\right|_{r=0}=0 \text { and }-\left.\lambda \frac{\partial T}{\partial r}\right|_{r=R}=0 \\
& \left.\lambda \frac{\partial T}{\partial z}\right|_{z=0}=q_{F} \text { and }-\left.\lambda \frac{\partial T}{\partial z}\right|_{z=e}=q_{R}
\end{aligned}
$$

with:

$$
q_{F}=h_{E Q}^{F}\left(T_{F}-T_{\infty}\right)+q_{d} \quad \text { and } \quad q_{R}=h_{E Q}^{R}\left(T_{R}-T_{\infty}\right)
$$

$h_{E Q}^{\beta}$ corresponds to the losses coefficient which gathers both convective and radiative losses $(\beta=R, F), q_{d}$ is the heat flux removed from the plate by the droplet stream, $T_{\infty}, T_{F}, T_{R}$ the surrounding, front and rear temperatures respectively. Laplace $\left({ }^{-}\right)$and Hankel $\left({ }^{\sim}\right)$ transforms are performed so that Eq. (6) can be solved:

$$
\frac{\partial \tilde{\bar{\theta}}_{n}^{2}}{\partial z}-\left(\alpha_{n}^{2}+\frac{p}{a}\right) \tilde{\bar{\theta}}_{n}=0
$$

where $\theta=T-T_{\text {init }}, p$ is the Laplace parameter and $\alpha_{n}=u_{n} / R$ and $u_{n}$ solutions of $J_{1}\left(u_{n}\right)=0$. Finally, the quadrupole method [20] yields a linear relationship between the rear face temperature $(z=e)$ and the cooling heat flux $(z=0)$ :

$$
\tilde{\bar{\theta}}_{n}^{R}=-\tilde{\bar{Z}}_{n}^{A}\left(p+a \alpha_{n}^{2}\right) \tilde{\bar{q}}_{n, d}+\tilde{\bar{Z}}_{n}^{B}\left(p+a \alpha_{n}^{2}\right) \frac{\tilde{\theta}_{n, \infty}}{p}
$$

where

$$
\tilde{\bar{Z}}_{n}^{A}(p)^{-1}=\left(h_{E Q}^{R}+h_{E Q}^{F}\right) \cosh \left(e \sqrt{\frac{p}{a}}\right)+\frac{h_{E Q}^{R} h_{E Q}^{F}}{\lambda \sqrt{\frac{p}{a}}} \sinh \left(e \sqrt{\frac{p}{a}}\right)+\lambda \sqrt{\frac{p}{a}} \sinh \left(e \sqrt{\frac{p}{a}}\right)
$$

and

$$
\tilde{\bar{Z}}_{n}^{B}(p)=\left(h_{E Q}^{F}+h_{E Q}^{R} \cosh \left(e \sqrt{\frac{p}{a}}\right)+\frac{h_{E Q}^{R} h_{E Q}^{F}}{\lambda \sqrt{\frac{p}{a}}} \sinh \left(e \sqrt{\frac{p}{a}}\right)\right) \tilde{\bar{Z}}_{n}^{A}(p)
$$

Laplace inversion of Eq. (8) makes a convolution product appears (noted ‘ $*$ '):

$$
\tilde{\theta}_{n}^{R}(t)=-\left[e^{-a \alpha_{n}^{2} t} \tilde{Z}_{n}^{A}(t)\right] * \tilde{q}_{n, d}(t)+\left[\int_{0}^{t} e^{-a \alpha_{n}^{2} t^{\prime}} \tilde{Z}_{n}^{B}\left(t^{\prime}\right) \mathrm{d} t^{\prime}\right] \tilde{\theta}_{n, \infty}
$$


The real temperature in time-space domain can be finally obtained through the Hankel inversion of the previous equations:

$$
\theta^{R}(r, e, t)=\frac{2}{R^{2}} \sum_{n=0}^{\infty} \tilde{\theta}_{n}^{R}(e, t) \frac{J_{0}\left(\alpha_{n} r\right)}{J_{0}^{2}\left(\alpha_{n} R\right)}
$$

\subsection{Inverse heat transfer problem}

Inverse heat conduction problem (IHCP) consists in using discrete temperature measurements inside a solid or at one of its external boundary in order to recover a time and/or space boundary condition (in our case the distribution of cooling flux at the droplet impact). This estimation problem is not well-posed, which means that low magnitude perturbation in the temperature measurement (noisy temperature) can generate large deviations on the estimated wall heat flux. This is due to the discrete features of measurements while the information that is looked for is a continuous function of time and/or space. We used the least square method as a regularization technique to overcome this effect and to efficiently stabilize the inversion algorithm. In our case, it can be easily solved because the solution of the direct heat transfer problem is known (see Eqs. (9) and (10)).

Eq. (9), expressed in Hankel domain ( ${ }^{\sim}$ ), is the starting point of the inverse heat conduction problem (IHCP); the integral form can be expressed using a truncature:

$$
\tilde{\theta}_{n}\left(t_{k}\right)=\sum_{j=1}^{k} e^{-a \alpha_{n}^{2}(k-j) \Delta t} \tilde{Z}_{n}^{A}((k-j) \Delta t) \tilde{q}_{n}\left(t_{j}\right) \Delta t=\sum_{l=1}^{k} S_{k j} \tilde{q}_{n}\left(t_{j}\right)
$$

where $\Delta t$ is the time step of the infrared camera (or a multiple), $\tilde{\theta}_{n}$ accounts for the initial field and $t_{k}=k \Delta t, t_{l}=l \Delta t$. The estimated harmonics of heat flux (noted '^') can be thus obtained using a least square method. In our case, a Gauss-Markov estimator was used to firstly estimate the temperature in the Hankel domain and to account of a non-uniform noise variance (i.e. the input temperature field is obtained from an averaging of the initial field from Cartesian to cylindrical frame, see the next section):

$$
\tilde{\theta}_{n}=\left(X_{n}^{t} \Omega X_{n}\right)^{-1} \Omega X_{n}^{t} \theta \quad \text { with } \Omega=\left[\begin{array}{cccc}
\sigma_{1}^{2} & 0 & 0 & 0 \\
0 & . & 0 & 0 \\
0 & 0 & . & 0 \\
0 & 0 & 0 & \sigma_{k_{\max }}^{2}
\end{array}\right]
$$

with $X_{l n}=\frac{2}{R^{2}} \frac{J_{0}\left(\alpha_{n} r_{l}\right)}{J_{0}^{2}\left(\alpha_{n} R\right)}$.

$$
\begin{aligned}
& \tilde{\theta}_{n}=\left[\begin{array}{c}
\tilde{\theta}_{n}\left(t_{1}\right) \\
\cdot \\
\cdot \\
\tilde{\theta}_{n}\left(t_{k_{\max }}\right)
\end{array}\right]=\mathbf{S}_{k}^{n} \tilde{\mathbf{q}}_{n}=\left[\begin{array}{cccc}
S_{11}^{n} & 0 & 0 & 0 \\
\cdot & \cdot & 0 & 0 \\
\cdot & \cdot & \cdot & 0 \\
S_{k_{\max }}^{n} & \cdot & \cdot & S_{k_{\max } k_{\max }}^{n}
\end{array}\right]\left[\begin{array}{c}
\tilde{q}_{n}\left(t_{1}\right) \\
\cdot \\
\cdot \\
\tilde{q}_{n}\left(t_{k_{\max }}\right)
\end{array}\right] \\
& \hat{\tilde{q}}_{n}=\left(S_{n}^{t} S_{n}\right)^{-1} S_{n}^{t} \hat{\tilde{\theta}}_{n}
\end{aligned}
$$

where $S_{n}$ are the sensity coefficient exactly calculated using the truncature expression (11) and Laplace inversion of thermal impedance namely $\tilde{Z}_{n}^{A}$. The return to time-space domain yields:

$$
q_{D C}\left(r, t_{k}\right)=\frac{2}{R^{2}} \sum_{n=0}^{n_{\max }} \frac{J_{0}\left(\alpha_{n} r\right)}{J_{0}^{2}\left(\alpha_{n} R\right)} \hat{\tilde{q}}_{n}\left(t_{k}\right)
$$

\subsection{Heat flux estimation from real temperature data}

The temperature $T(x, y, t)$ obtained from the infrared camera is available in a Cartesian reference frame while the input data $T(r, t)$ of the model must be known in a cylindrical reference frame. Thus, it requires the conversion and the averaging of the initial Cartesian temperature field in order to obtain the input data. As a consequence, we cannot access to the real heat flux $q_{D C}(x, y, t)$ but only to an averaged heat flux $q_{D C}(r, t)$. So, the first step of the inversion procedure is to obtain the input temperature by averaging the initial field. In a second step, the Hankel transform ( ${ }^{\sim}$ ) of the temperature $\tilde{\theta}_{n}$ will be estimated using a Gauss-Markov estimator (Eq. (12)) because the temperature distribution $T(r, t)$ shall be subject to a nonuniform noise variance. Then, the harmonics of heat flux (in the Hankel domain) can be estimated using Eq. (14). As the cooling flux is very weak, we must consider all the heat loss by conduction, convection and radiation. Those are estimated using a relaxation run during which no droplet stream impinges the hot slab $[21,22]$. 


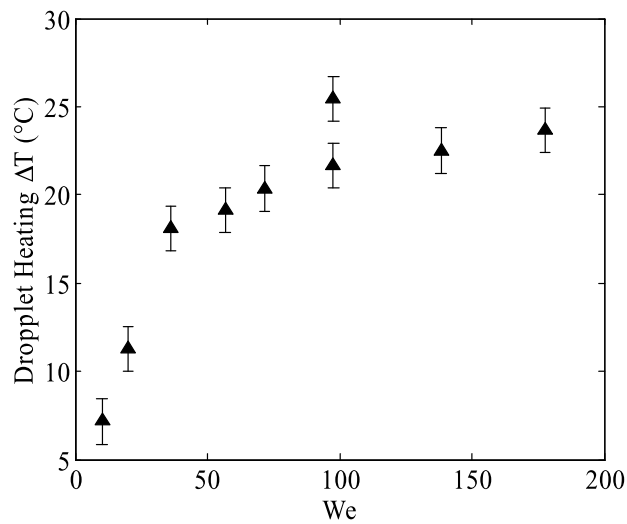

Fig. 5. Liquid increase in temperature during the impingement onto the wall as a function of the normal Weber number We $e_{n}$ of the incident droplets $\left(D=137 \mu \mathrm{m}, T_{w}=540^{\circ} \mathrm{C}, f=12 \mathrm{kHz}\right)$.

\section{Results and discussion}

Measurements were carried out for three different sizes of droplets ( $80 \mu \mathrm{m}, 137 \mu \mathrm{m}$ and $180 \mu \mathrm{m}$ ). The impact angle of the droplet stream was modified step by step from $10^{\circ}$ to $80^{\circ}$ while the injection velocity was fixed at about $10 \mathrm{~m} / \mathrm{s}$. The case of normal impacts was not considered because of the need to discriminate between primary and secondary droplets that may also coalesce in this configuration. The temperature of the liquid in the injector body remained at $24{ }^{\circ} \mathrm{C}$ throughout the experiment.

\subsection{Liquid phase heating}

Fig. 5 shows the liquid temperature increase as a function of the Weber number, which was estimated using the normal component of the droplet velocity $\left(W e_{n}=\rho_{l} V_{n}^{2} D / \gamma\right)$ in the case of the $137 \mu \mathrm{m}$ droplets. When $W e_{n}<60$, the impact regime is a rebound, whereas the regime observed beyond this value of the Weber number is a splashing. It can be noticed that the heating of the droplets is all the more important than the Weber number is high. The droplet variation in temperature increases progressively with the Weber number, there is no discontinuity between the bouncing and the splashing regimes.

For the lowest values of $W e_{n}$, the droplet heating rises rapidly with $W e_{n}$ and reaches a plateau in the beginning of the splashing region. The same evolution was already observed in [17]. In this study, the heating was about $10^{\circ} \mathrm{C}$ higher. However, the experimental conditions were different, especially the nickel sample was much thicker and the frequency of the droplet injection was smaller. It is also worth to mention that there is a certain scattering of the measurements represented in the figure by error bars. This scattering is not only due to measurement inaccuracies. Measurement conditions can be more or less stable in particular in the bouncing regime and the wall surface can be altered by a thermal fatigue during the measurements.

Fig. 6 shows the evolution of the droplet increase in temperature for the three different droplet sizes as a function of the Mundo number $K$. This number is a combination between the Weber number and the Ohnesorge number.

$$
K=W e_{n} O h^{0.4} \text { with } O h=\mu_{l} / \sqrt{\rho_{l} D_{d} \gamma}
$$

$K$ was introduced first by Mundo [19] and was used successfully by Cossali et al. [5] to describe the transition between the deposition and the splashing regime. Several studies suggest that the transition between the splashing and the rebound regimes is situated around a constant value of $K$ equal to 400 . This parameter is therefore particularly adapted to compare the heating of droplet having different sizes and velocities. The results in Fig. 6 suggest that the heating of the droplets is comparable whatever the size of the droplets in the splashing. In contrast, at a given value of $K$, the heating seems more important for the droplets of smaller sizes in the regime of rebound.

\subsection{Wall cooling}

The temperature at the rear face of the nickel sample is recorded during the cooling once the heating by the inductor is switched off. The temperature is recorded two times by the IR camera, one time during the cooling of the sample in the presence of droplets and a second time during the same cooling in the absence of droplets. After inversion, the wall heat flux obtained for the cooling with droplet is subtracted from the heat flux measured without droplet at the same wall temperature. This subtraction allows removing the heat losses associated with the free convection, the heat conduction within the sample holder, and the radiative transfer to the surroundings. Finally the result is divided by the injection frequency, which allows determining $\Phi_{w}$ the heat removed from the wall by a single droplet. 


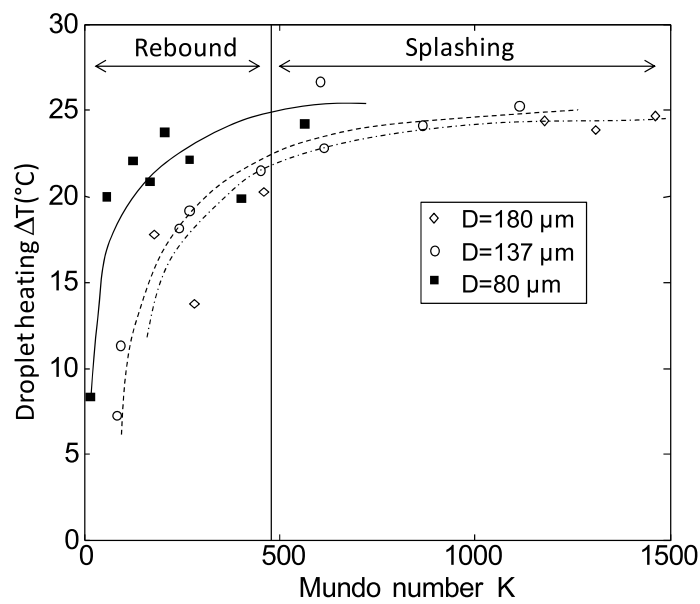

Fig. 6. Evolution of the droplet increase in temperature as a function of the Mundo number $K$. $\left(T_{w}=540^{\circ} \mathrm{C}\right.$, measurements obtained at a fixed injection frequency $f=12 \mathrm{kHz}$.)

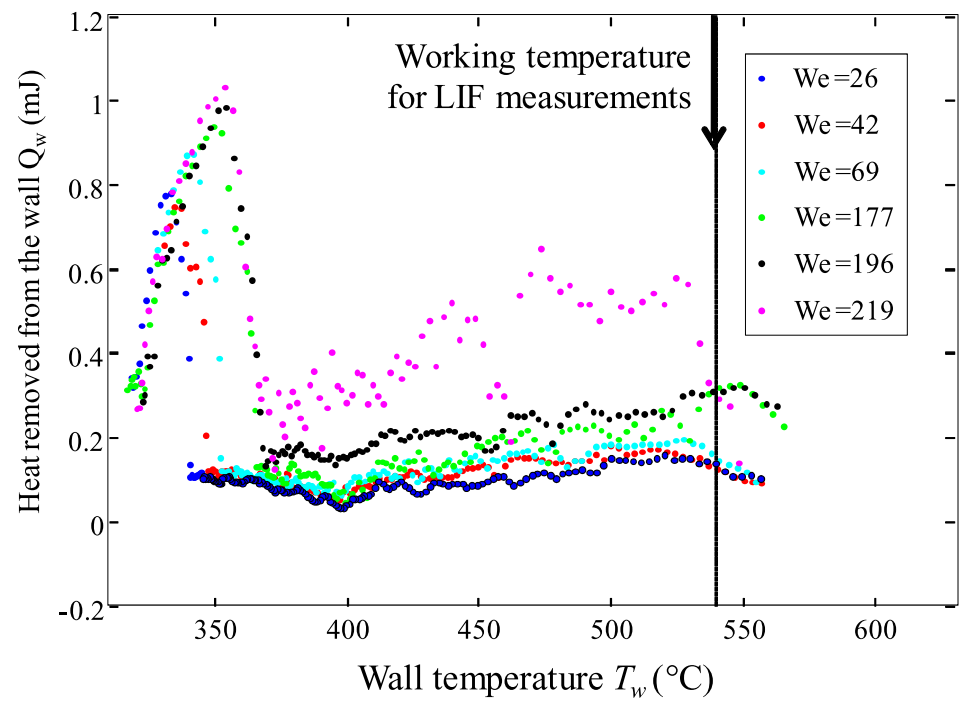

Fig. 7. Heat removed by a single droplet as a function of the wall temperature $(D=180 \mu \mathrm{m}, f=12 \mathrm{kHz})$.

Fig. 7 shows the evolution of $\Phi_{w}$ as a function the wall temperature $T_{w}$ for a droplet size of $180 \mu \mathrm{m}$. As expected, the heat removed from the wall increases with the wall temperature in the regime of film boiling and it is the lowest when the wall temperature is equal to the Leidenfrost temperature. At the end of the cooling, the wall temperature decreases below the Leidenfrost temperature and the liquid can rewet the wall. $\Phi_{w}$ is then sharply enhanced. Comparison of the curves in Fig. 7 shows a gradual increase of the Leidenfrost temperature with the Weber number of the incident droplets. The Leidenfrost temperature of a sessile droplet (static droplet) is even smaller as it is about $250{ }^{\circ} \mathrm{C}$. Moreover, it appears that the higher the Weber number is, the more important is the cooling rate of the wall.

Fig. 8 shows the cooling efficiency $\varepsilon$ as a function of the normal Weber number for the different droplet sizes at a fixed wall temperature. The cooling efficiency $\varepsilon$ is defined as:

$$
\varepsilon=\Phi_{w} /\left[m\left(L_{v}+C p_{l}\left(T_{b}-T_{i n j}\right)\right)\right]
$$

where $T_{b}$ is the boiling temperature of water and $T_{i n j}$ the injection temperature. It is apparent that the cooling efficiency is all the more important than the droplet size is small. This result has been already observed in several studies including Bernardin and Mudawar [7] where the cooling by monodisperse droplets streams is also investigated. When the droplets are getting smaller, the ratio surface/volume increases which tend to enhance the transfers, in particular the heat flux related to the evaporation as it will be described in the following. The increase of the normal component of the droplet velocity at a given droplet size improves also the heat exchange process as illustrated in Fig. 7. 


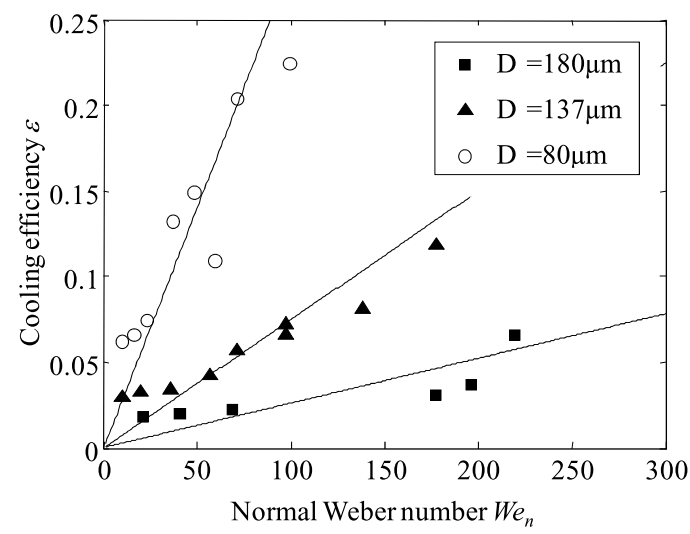

Fig. 8. Efficiency of the wall cooling as a function of the normal Weber number $\left(T_{\text {wall }}=540^{\circ} \mathrm{C}, f=12 \mathrm{kHz}\right)$.

\subsection{Energy balance of the droplet/wall interaction}

A simplified expression of the energy balance of the heat fluxes involved in the droplet/wall interactions can be written as follows:

$$
\Phi_{w}=Q_{l}+\Delta m \cdot\left(L_{v}+C p_{v} \Delta T_{v}\right)
$$

In this expression, $Q_{l}$ corresponds to the sensible heat gained by the liquid:

$$
Q_{l}=\left[(m-\Delta m) C p_{l} \Delta T_{l}+\Delta m C p_{l}\left(T_{s}-T_{l, 0}\right)\right]
$$

In these expressions, $\Delta m$ is the vapor mass of the droplet evaporated during its impingement. $\Delta T_{l}$ is the increase in the liquid temperature measured by the two-color LIF thermometry. $T_{l, 0}$ is the liquid temperature before the impingement. $T_{s}$ is the surface temperature at which the liquid evaporation occurs. $C p_{v} \Delta T_{v}$ is the sensible heat taken by the vapor which has a temperature that is increased from $T_{S}$ at the droplet surface. $T_{S}$ and $\Delta T_{v}$ are not measured but in first approximation $T_{S}$ can be replaced by the boiling temperature of the liquid and $\Delta T_{v}$ can be overestimated assuming $\Delta T_{v}=T_{w b}=T_{w}-T_{b}$. A model for the thickness of the vapor film squeezed between the wall and the droplet, and for the flow field within this vapor film would be required to estimate more accurately the sensible heat $C p_{v} \Delta T_{v}$ gained by the vapor and $T_{s}$. Introducing the Jakob number $J a=C p_{v} T_{w b} / L_{v}$ :

$$
\Phi_{w}=Q_{l}+\Phi_{v a p} \cdot(1+J a)
$$

with

$$
\Phi_{v a p}=L_{v} \Delta m
$$

Finally,

$$
\Delta m=\frac{\Phi_{w}-m C p_{l} \Delta T_{l}}{L_{v}(1+J a)+C p_{l}\left(T_{b}-T_{l, f}\right)}
$$

Fig. 9 shows the evolution of $\Delta m / m$ as function of the normal Weber number $W e_{n}$. It can be observed that this parameter varies strongly with the droplet size. For the smallest droplets $(D=80 \mu \mathrm{m})$, the relative variation of the droplet mass is significant as it can reach about $25 \%$, while it is negligible for the largest droplets. Negative values for $\Delta m$ are sometimes found using Eq. (22), when $m C p_{l} \Delta T_{l}$ has a slightly higher value than $\Phi_{w}$ due the measurements uncertainties. As expected, $\Delta m / m$ is increasing with the Weber number and this dependency seems to be all the more important than the droplet is small.

Finally, the contribution of the sensitive heating of the liquid to the cooling appears to increase with the droplet size and to decrease with the normal Weber number $W e_{n}$ as shown in Fig. 10 . The ratio $\eta=Q_{l} / \Phi_{w}$ is slightly above 1 for $D=180 \mu \mathrm{m}$, which can be related to the measurement uncertainties. This result is interesting for the modeling of spray cooling. It points out that the interest of taking into account the sensible heat gained by the liquid depends firstly on the droplet sizes and secondly on the Weber number. The heat flux related to evaporation is predominant in the cooling by the smaller droplets, which makes the cooling by these droplets more efficient. For the larger droplets, the evaporation is very moderate (Fig. 9) and the sensible heat gained by the liquid contributes roughly to the whole cooling of the wall (Fig. 10). 


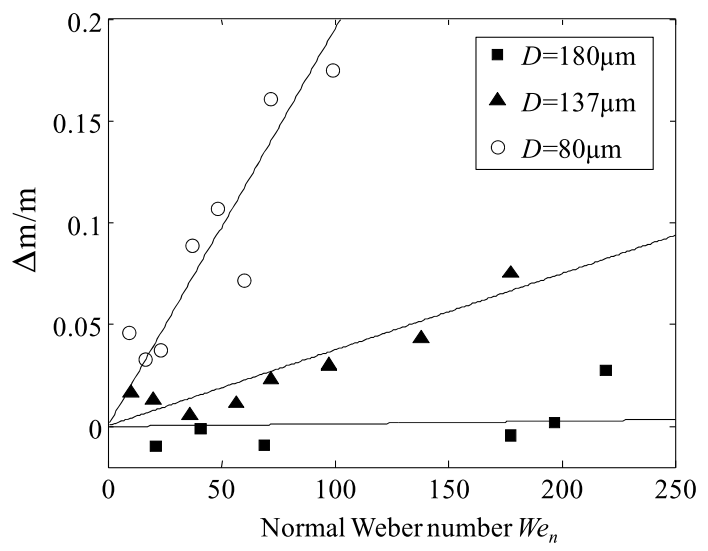

Fig. 9. Evolution of $\Delta m / m$ as a function of the normal Weber number $\left(T_{\text {wall }}=540^{\circ} \mathrm{C}, f=12 \mathrm{kHz}\right)$.

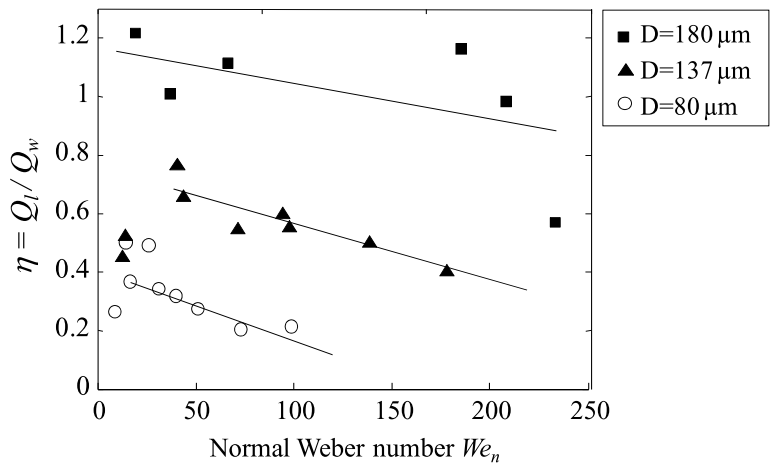

Fig. 10. Evolution of $\eta$ as a function of the normal Weber number $\left(T_{\text {wall }}=540^{\circ} \mathrm{C}, f=12 \mathrm{kHz}\right)$.

\section{Conclusion}

This article presents an experimental study of the heat transfers that occur during the impact of droplets in the film boiling regime. Different measurement techniques are used to characterize the transfers on the sides of the liquid phase and the solid surface. Infrared thermography combined with an inverse heat conduction model allows estimating the heat flux removed from the wall. 2cLIF thermometry is used to measure the temperature increase of the liquid and thus to determine the sensible heat gained by the liquid during the droplet impingement. The comparison of these heat fluxes allows estimating the contribution of the evaporation in the cooling of the wall. The interest of this approach is that it is almost impossible to quantify directly the mass of liquid in the droplets because they can be strongly deformed at the contact with the wall. However, for a better assessment of the evaporated mass it could be interesting to quantify the gain of enthalpy of the vapor trapped in the vapor film squeezed between the droplet and the wall. Nevertheless, the results reveal also that the contribution of the sensible heat gained by the liquid to the wall cooling decreases strongly with the droplet size. The heat flux related to evaporation is predominant in the cooling by the smaller droplets, which makes the cooling by the smaller droplets more efficient.

\section{Nomenclature}

\section{Acknowledgements}

This work has been supported by the French National Agency (ANR) in the frame of the research program IDHEAS (No. ANR-NT09 432160).

\section{References}

[1] M. Rein, Interactions between drops and hot surfaces, in: M. Rein (Ed.), Drop-Surface Interactions, Springer, Wien, New York, 2002.

[2] R. Rioboo, C. Tropea, M. Marengo, Outcomes from a drop impact on solid surfaces, Atomization and Sprays 11 (2) (2001) $155-165$.

[3] S.C. Yao, K.Y. Cai, The dynamics and Leidenfrost temperature of drops impacting on a hot surface a small angles, Experimental Thermal and Fluid Science 1 (1988) 363-371. 
[4] S. Sikalo, M. Marengo, C. Tropea, E.N. Ganic, Analysis of impact of droplets on horizontal surfaces, Experimental Thermal and Fluid Science 25 (2002) 503-510.

[5] G.E. Cossali, M. Marengo, M. Santini, S. Fest, Effect of wall effusivity on thermally induced secondary atomisation of single drop impacting onto a tilted surface, in: 10th International Conference on Liquid Atomization and Spray System, Kyoto, Japan, 2006.

[6] N. García Rosa, P. Villedieu, J. Dewitte, G. Lavergne, A new droplet-wall interaction model, in: 10th International Conference on Liquid Atomization and Spray System, Tokyo, Japan, 2006.

[7] J.D. Bernardin, I. Mudawar, Film boiling heat transfer of droplet streams and sprays, International Journal of Heat and Mass Transfer 40 (2) (1997) 2579-2593.

[8] K.J. Baumeister, J.J. Simon, Leidenfrost temperature - its correlation for liquid metals, cryogens, hydrocarbons and water, Transactions of the ASME: Journal of Heat Transfer 95 (1973) 166-173.

[9] P. Le Clercq, Contribution à l'étude expérimentale et théorique des interactions gouttes/paroi, PhD thesis of Ecole Nationale de l'Aéronautique et de l'Espace, 2000.

[10] N.G. Rosa, P. Villedieu, J. Dewitte, G. Lavergne, A new droplet-wall interaction model, in: 10th International Conference on Liquid Atomization and Spray Systems (ICLASS-2006), Kyoto, 2006.

[11] L.H.J. Wachters, N.A.J. Westerling, The heat transfer from a hot wall to impinging water drops in the spheroidal state, Chemical Engineering Science 21 (11) (1966) 1047-1056.

[12] D.B. Reid, An algorithm for tracking multiple targets, IEEE Transactions on Automatic Control AC-24 (1979) 843-854.

[13] S. Boulesteix, Cisaillement d'une interface gaz-liquide en conduite et entraînement de gouttelettes, 2010.

[14] G. Castanet, P. Lavieille, M. Lebouche, F. Lemoine, Measurement of the temperature distribution within monodisperse combusting droplets in linear streams using two-color laser-induced fluorescence, Experiments in Fluids 35 (6) (2003) 563-571.

[15] V. Deprédurand, G. Castanet, F. Lemoine, Heat and mass transfer in evaporating droplets in interaction: influence of the fuel, International Journal of Heat and Mass Transfer 53 (17-18) (2010) 3495-3502.

[16] G. Castanet, T. Liénart, F. Lemoine, Dynamics and temperature of droplets impacting onto a heated wall, International Journal of Heat and Mass Transfer 52 (3-4) (2009) 670-679.

[17] P. Dunand, G. Castanet, F. Lemoine, A two-color planar LIF technique to visualize the temperature of droplets impinging onto a heated wall, in: 15th International Symposium on Applications of Laser Techniques to Fluid Mechanics, Lisbon, Portugal, 2010, pp. 05-08.

[18] P. Lavieille, A. Delconte, D. Blondel, M. Lebouché, F. Lemoine, Non-intrusive temperature measurements using three-color laser-induced fluorescence, Experiments in Fluids 36 (5) (2004) 706-716.

[19] A. Labergue, A. Delconte, G. Castanet, F. Lemoine, Study of the droplet size effect coupled with the laser light scattering in sprays for two-color LIF thermometry measurements, Experiments in Fluids 52 (5) (2012) 1121-1132.

[20] D. Maillet, S. André, J.C. Batsale, A. Degiovanni, C. Moyne, Thermal Quadrupoles: Solving the Heat Equation Through Integral Transforms, John Wiley \& Sons, 2000.

[21] M. Gradeck, F. Lelong, N. Seiler, D. Maillet, How to estimate the heat transfer due to droplets impinging onto a hot slab?, in: ExHFT-7, Krakow, Poland, 2009.

[22] D. Maillet, M. Gradeck, B. Rémy, A. Ouattara, F. Lelong, Inverse conduction technique in Hankel domain using infrared thermography: application to water jet or droplet stream quenching a metal disk, in: 14th International Heat Transfer Conference, Washington, DC, 2010. 